\title{
Dynamics of trade-by-trade price movements: decomposition and models
}

\author{
Tina HviId RydBerg \\ BNP Paribas, 10 Harewood Avenue, NW1 6AA London, UK. \\ tina.rydberg@paribas.com \\ NeIL SHEPHARD \\ Nuffield College, Oxford OX1 1NF, UK. \\ neil.shephard@nuf.ox.ac.uk
}

January 1, 2002

\begin{abstract}
In this paper we introduce a decomposition of the joint distribution of price changes of assets recorded trade-by-trade. Our decomposition means that we can model the dynamics of price changes using quite simple and interpretable models which are easily extended in a great number of directions, including using durations and volume as explanatory variables. Thus we provide an econometric basis for empirical work on market microstructure using time series of transactions data.

We use maximum likelihood estimation and testing methods to assess the fit of the model to a year of IBM stock price data taken from the New York Stock Exchange.

Keywords: Activity, autologistic, conditional independence, decomposition, directions, durations, forecasting, GLARMA, logarithmic distribution, prediction decomposition, size, transactions data.
\end{abstract}




\section{Introduction}

Let $p(u)$ denote the price at time $u$ of the most recent transaction of an asset. Here $u$ is a continuous clock, but prices are only updated when a trade actually occurs. By construction the price can be written as

$$
p(u)=p(0)+\sum_{t=1}^{N(u)} Z_{t}
$$

where $N(u)$ is the number of trades recorded in the interval from time 0 up until time $u$ and $Z_{t}$ is the price movement associated with the $t-t h$ trade. In practice many of the $Z_{t}$ are exactly zero. Rydberg and Shephard (2000) and Rogers and Zane (1998) use this framework to study the evolution of transaction prices. They model $N(u)$ as a counting process with new arrivals being generated by a Cox process, that is a Poisson process with a random intensity. They are unspecific about the $Z_{t}$ process beyond the use of simple descriptive Markov chains. Some of the econometric issues which arise with unequally spaced financial data are discussed at length in Engle and Russell (1998), Engle (2000) and Engle and Russell (2002).

In this paper we model the joint distribution of price movements $Z_{t}$, focusing on the econometric problem that the price movements are restricted to take on integer multiples of a smallest non-zero price change, i.e. a tick. The tick size depends on the institutional setting. When normed this means price movements can be thought of as being integers. To start with we will model the $Z_{t}$ as being dependent only on themselves. The problem of modelling using larger filtrations is dealt with later in the paper. Then let $Z_{t} \in I$ be an integer process and $\mathcal{F}_{t}=\sigma\left(Z_{s}: s \leq t\right)$ be its natural filtration. We will be primarily interested in the joint distribution of the movements which are given by

$$
\operatorname{Pr}\left(Z_{1}, \ldots, Z_{n} \mid \mathcal{F}_{0}\right)=\prod_{t=1}^{n} \operatorname{Pr}\left(Z_{t} \mid \mathcal{F}_{t-1}\right),
$$

using a prediction decomposition. The focus will be on specifying $\operatorname{Pr}\left(Z_{t} \mid \mathcal{F}_{t-1}\right)$.

The main contribution of this paper is to suggest decomposing $Z_{t}$ into three components - "activity", "direction" and "size". The variables measure, respectively: (i) if the price moved, (ii) which direction did it move, (iii) how far did it move. Our claim will be that this structure allows a relatively simple econometric analysis of sequential price movements.

There already exists a literature on the modelling of trade-by-trade price dynamics. Russell and Engle (1998) suggest using a conditional multinomial model for specifying 
$\operatorname{Pr}\left(Z_{t} \mid \mathcal{F}_{t-1}\right)$, in a sense generalising previous work of Hausman, Lo, and MacKinlay (1992) on probit models for transaction data. Hasbrouck (1999) builds a class of dynamic latent variable models for efficient prices and traders' cost which uses an economically motivated truncation to force prices to live on a lattice. The general issue of discreteness of asset prices is discussed at length by Campbell, Lo, and MacKinlay (1997, pp. 98-144).

Other work which relates to this topic includes papers by Meddahi, Renault, and Werker (1998), Ghysels and Jasiak (1998) and Manganelli (2001) who combine GARCH models for price returns with ACD style models for the times between trades. In this context this work has the disadvantage that their price process does not live on the required lattice observed in the data. The paper by Darolles, Gourieroux, and Le Fol (2000), which we first saw after the circulation of the first draft of this paper, is much closer to the framework of Rydberg and Shephard (2000) and Rogers and Zane (1998). Darolles, Gourieroux, and Le Fol (2000) use the structure (1) but assume the $Z_{t}$ process is Markov living on the points $-1,0,1$. This modelling assumption is combined with a reduction in the dataset by using quote data to allow them only to model buys from the market maker - which in turn makes the assumption that the price movements are at most one tick in absolute value more realistic.

Our paper has the following basic structure. In Section 2 we introduce our decomposition of the price movements. Section 3 will look at our initial empirical models for the activity, direction and size of price movements - taken together these three models yield an overall model of price movements. Section 4 places our suggestion in the context of the literature, as well as suggesting various extensions of the basic model construct. Section 5 concludes.

\section{Decomposition of price movements}

Potentially the distribution of $Z_{t} \mid \mathcal{F}_{t-1}$ can be quite complicated. Our approach is to break down the pieces of $Z_{t}$ into bits and then model these sequentially. Note that there is no loss of information in this decomposition.

To carry out our decomposition define the $t-t h$ price move as

$$
Z_{t}=A_{t} D_{t} S_{t}
$$

We will let $A_{t}$ take on only two values: 0,1 . When $A_{t}=0$, we define for notational convenience (there is no loss in doing this), $D_{t}=S_{t}=0$. Otherwise, when $A_{t}=1$ we let 
$D_{t}$ and $S_{t}$ live on the structure:

$$
D_{t}=-1,1 \quad \text { and } \quad S_{t}=1,2, \ldots
$$

Thus we have that if $A_{t}$ is zero then $Z_{t}$ must be zero. This means the price does not move or, in other words, is In-Active. If $A_{t}=1$ then there are Active price movements. The non-zero price movement must be $Z_{t}=D_{t} S_{t}$. Likewise, if we assume $A_{t}=1$, then $D_{t}$ controls the Direction of the price move. If $D_{t}=1$ the price moves upwards, else it moves downwards. Finally, $S_{t}$ controls the Size of price movements. This suggests the decomposition of price movements into

$$
\operatorname{Pr}\left(Z_{t}=0 \mid \mathcal{F}_{t-1}\right)=\operatorname{Pr}\left(A_{t}=0 \mid \mathcal{F}_{t-1}\right)
$$

while for $z_{t} \neq 0$

$$
\begin{aligned}
\operatorname{Pr}\left(Z_{t}=\right. & \left.z_{t} \mid \mathcal{F}_{t-1}\right)=\operatorname{Pr}\left(A_{t}=1 \mid \mathcal{F}_{t-1}\right) \\
& \times\left\{\begin{array}{c}
\operatorname{Pr}\left(S_{t}=z_{t} \mid \mathcal{F}_{t-1}, A_{t}=1, D_{t}=1\right) \operatorname{Pr}\left(D_{t}=1 \mid \mathcal{F}_{t-1}, A_{t}=1\right)+ \\
\operatorname{Pr}\left(S_{t}=-z_{t} \mid \mathcal{F}_{t-1}, A_{t}=1, D_{t}=-1\right) \operatorname{Pr}\left(D_{t}=-1 \mid \mathcal{F}_{t-1}, A_{t}=1\right)
\end{array}\right\}
\end{aligned}
$$

The implication of this decomposition is that there are exactly three pieces of modelling to carry out

- $\operatorname{Pr}\left(A_{t} \mid \mathcal{F}_{t-1}\right)$ - a binary process on $\{0,1\}$ modelling activity (the price moves or not).

- $\operatorname{Pr}\left(D_{t} \mid \mathcal{F}_{t-1}, A_{t}=1\right)$ - another binary process on $\{-1,1\}$ modelling the direction of the price moves.

- $\operatorname{Pr}\left(S_{t} \mid \mathcal{F}_{t-1}, A_{t}=1, D_{t}\right)$ - a process on the strictly positive integers modelling the size of price moves.

Potentially each of these models has to be constructed separately — basing each on the complete history of the $Z_{t}$ process. Although this sounds a difficult task we will see that our estimated specifications will have very simple interpretable structures which do not immediately appear when we model the $Z_{t}$ directly. It will be helpful to decompose the natural filtration $\mathcal{F}_{t}$ into its constituent parts $-\mathcal{F}_{t}^{A}=\sigma\left(A_{s}: s \leq t\right), \mathcal{F}_{t}^{D}=\sigma\left(D_{s}: s \leq t\right)$ and $\mathcal{F}_{t}^{S}=\sigma\left(S_{s}: s \leq t\right)$. Of course $\mathcal{F}_{t}=\mathcal{F}_{t}^{A, D, S}$.

Finally, before we detail the modelling of activity, direction and size of the price movements we should note that although we can model these processes separately we are specifying a multivariate model. Hence in principle we cannot simulate a sequence of 
activities using just $\operatorname{Pr}\left(A_{t} \mid \mathcal{F}_{t-1}\right)$ as we need all three models to simulate past values of $Z_{t}$. Thus we are not specifying a marginal model for the processes for activities, directions or sizes. An implication of this is that a structural break in any of the three processes $A_{t}\left|\mathcal{F}_{t-1}, D_{t}\right| \mathcal{F}_{t-1}, A_{t}=1$ and $S_{t} \mid \mathcal{F}_{t-1}, A_{t}=1, D_{t}$ will imply a structural break in the joint process.

\section{Preliminary models for the components}

\subsection{The data}

To start our empirical modelling we will work with the natural filtration of the price movements, building an initial empirical model for $\operatorname{Pr}\left(Z_{t} \mid \mathcal{F}_{t-1}\right)$ via the construction of three models: those for activity, direction and size. The next section will extend this work to allow us to condition on a wider filtration. The trade data used in this paper is for the IBM stock recorded electronically at the New York Stock Exchange in 1995. We first construct a time series for each day on which the exchange was open, computing the price changes at each trade (rescaling the data to have a tick size of one). We then have deleted the first 15 minutes of every day. This is to avoid having to deal with the effects of the call auction which takes place in the morning to set off the trading. We also cut out all trades registered after 16.00 as this is the official closing of the exchange and our initial data analysis suggested the data was significantly different when it had a time stamp which was after 16.00 .

For this paper we constructed a single series by concatenating each of the above series (whose overnight effects were removed by deleting the action of removing the first 15 minutes of the day) for individual days. The length of the total data set when all

exchanges in the US are considered 413,906, this is too much data to initially handle and therefore we have limited out analysis to the trades performed at NYSE (trades coded with an N). We have also deleted all trades which have an error code. This leaves us with a total of 173, 146 observations to model. Of these 33,184 are non-zero (and so moved the price), which means the data we model for directions and size will only be $19 \%$ of the size of the activity series. Throughout we ignore the availability of quote data. 


\subsection{The activity of prices}

\subsubsection{Autologistic model}

Our initial parametric model for $\operatorname{Pr}\left(A_{t} \mid \mathcal{F}_{t-1}\right)$ will be an autologistic model based on $\mathcal{F}_{t}$. Recall for an autologistic we write

$$
\operatorname{Pr}\left(A_{t}=1 \mid \mathcal{F}_{t-1}\right)=p\left(\theta_{t}^{A}\right), \quad \text { where } \quad p\left(\theta_{t}^{A}\right)=\frac{\exp \left(\theta_{t}^{A}\right)}{1+\exp \left(\theta_{t}^{A}\right)}
$$

and

$$
\theta_{t}^{A}=x_{t}^{\prime} \beta+g_{t}, \quad \text { where } \quad g_{t}=\sum_{j=1}^{p} \beta_{j} A_{t-j}
$$

with $x_{t}$ being potential combinations and subsets of $\mathcal{F}_{t-1}$. This model structure was introduced by Cox (1958) (see Cox and Snell (1989) for an exposition) and has some significant advantages ${ }^{1}$. The log-likelihood for the autologistic is concave and so numerical optimisation is completely straightforward, allowing standard logistic regression software to be used to rapidly and reliably fit the model (e.g. McCullagh and Nelder (1989)).

We use a general-to-specific model selection approach (see, for example, Hendry (1995)), estimating a complete model and then testing down insignificant lags. To start off we only allow 20 lags of all of the variables $\left(A_{t}, D_{t}\right.$ and $\left.S_{t}\right)$ to enter the model. In practice, in order to reduce multicolinearity, it makes sense to transform the size variable $S_{t}$ into

$$
L_{t}=S_{t}-A_{t}
$$

which is zero unless $S_{t}$ is bigger than one. We call $L_{t}$ a large move variable. After the model is fitted we will look at a portmanteau test to see its ability to capture the main features of the data. This is based on the residuals

$$
u_{t}=\frac{A_{t}-p\left(\theta_{t}^{A}\right)}{\sqrt{p\left(\theta_{t}^{A}\right)\left\{1-p\left(\theta_{t}^{A}\right)\right\}}},
$$

which should be uncorrelated with zero mean and unit conditional (and unconditional) variance. The $\left\{u_{t}\right\}$ are then used inside a Box-Pierce statistic as a measure of residual dependence.

The result of the model fitting and diagnostic checking are shown in Table 1. At lag two $L_{t}$ is significant. It indicates that if there is a large movement in the market followed

\footnotetext{
${ }^{1} \mathrm{~A}$ standard latent variable interpretation of these models writes

$$
\operatorname{Pr}\left(Y_{t}=1 \mid X_{t}\right)=\operatorname{Pr}\left(\theta_{t}+U>0\right)=p\left(\theta_{t}\right),
$$

where $U$ has a logistic distribution with parameters $(0,1)$. Replacing the logistic distribution by a standard normal produces a probit model.
} 
by another trade, then there will be an increased probability of subsequent movements (of any size) in the price. That is large movements are associated with subsequent high volatility. The direction variables are negative, which suggests past falls in the prices tend to increase the chance that there will be a future movement in the market. This seems close to the leverage effect which is emphasised in the ARCH literature (e.g. Nelson (1991)).

Table 1 also indicates bid-ask bounce, for if the two lagged direction variables have opposite signs then the direction variable is damped down so reducing the chance of future price movements. However, this last effect will be clearer when we model the dynamics of the direction of price movements.

\begin{tabular}{|c|cc||c|cc|}
\hline Variable & Coef. & StR. Err. & Variable & Coef. & StR. Err. \\
\hline$A_{t-1}$ & 0.641 & 0.014 & $A_{t-9}$ & 0.078 & 0.016 \\
$D_{t-1}$ & -0.105 & 0.013 & $A_{t-10}$ & 0.049 & 0.016 \\
$A_{t-2}$ & 0.244 & 0.015 & $A_{t-11}$ & 0.066 & 0.016 \\
$L_{t-2}$ & 0.289 & 0.092 & $A_{t-12}$ & 0.069 & 0.016 \\
$D_{t-2}$ & -0.050 & 0.013 & $A_{t-13}$ & 0.041 & 0.016 \\
$A_{t-3}$ & 0.253 & 0.015 & $A_{t-14}$ & 0.090 & 0.016 \\
$A_{t-4}$ & 0.175 & 0.015 & $A_{t-15}$ & 0.050 & 0.016 \\
$A_{t-5}$ & 0.173 & 0.015 & $A_{t-16}$ & 0.036 & 0.016 \\
$A_{t-6}$ & 0.111 & 0.015 & $A_{t-17}$ & 0.035 & 0.016 \\
$A_{t-7}$ & 0.113 & 0.015 & $A_{t-18}$ & 0.039 & 0.016 \\
$A_{t-8}$ & 0.077 & 0.015 & $A_{t-19}$ & 0.059 & 0.016 \\
Const. & 1.958 & 0.012 & $A_{t-20}$ & 0.053 & 0.016 \\
\hline $\mathrm{Q}$ & $T \sum_{j=1}^{Q} r_{j}^{2}$ & & & Log-like $=-82,313$ \\
\hline 20 & 23.20 & $(31.41)$ & & & \\
100 & 698.4 & $(124.3)$ & & & \\
1500 & 5,489 & $(1591)$ & & & \\
\hline
\end{tabular}

Table 1: Estimation for the activity using an autologistic model. Std. Err. denotes the (uncorrected) standard deviation. $r_{j}$ denotes the series correlation coefficient at lag $j$ for the the standardised residuals $u_{t}$. The figures in brackets are corresponding 95 percentage points on the $\chi_{Q}^{2}$ distribution.

Finally, Table 1 shows the coefficients in front of the activity variables decay down — starting at 0.6 at lag one and falling to 0.2 at lag three. However, for longer lags the decay is quite slow and is not sufficiently well captured by our imposed artificial cutoff at lag 20. As a result the diagnostic checks on the residuals behave well at short lags, but poorly at longer lags as there is significant dependence at 1000s of lags in the activity variable. In order to model this parsimoniously we have to move away from autologistic 
models and into constructions which allow moving average type behaviour. This can be carried out by introducing GLARMA type models.

\subsubsection{GLARMA binary model}

We could generalise the autologistic structure (2) by allowing

$$
g_{t}=\sum_{j=1}^{p} \gamma_{j} g_{t-j}+\sum_{j=1}^{q} \delta_{j} A_{t-j}
$$

but this is typically numerically unstable and so difficult to work with. Shephard (1994) has studied a number of alternatives, which are called generalized linear autoregressive moving average (GLARMA) models. The one we favour here puts

$$
g_{t}=\sum_{j=1}^{p} \gamma_{j} g_{t-j}+\sigma v_{t}+\sigma \sum_{j=1}^{q} \delta_{j} v_{t-j}, \quad \sigma>0
$$

where

$$
v_{t}=\frac{\left\{A_{t-1}-p\left(\theta_{t-1}^{A}\right)\right\}}{\sqrt{p\left(\theta_{t-1}^{A}\right)\left\{1-p\left(\theta_{t-1}^{A}\right)\right\}}} .
$$

Importantly $\left\{v_{t}\right\}$ is a Martingale difference sequence with a unit conditional variance. This style of model is adopted in Russell and Engle (1998) in their multinomial construction.

GLARMA models have some of the properties of ARMA models. This follows as $\left\{v_{t}\right\}$ has a zero mean and unit conditional and unconditional variance. The implication of this is that $\left\{g_{t}\right\}$ is a linear ARMA process driven by a weak white noise error term. Hence it is covariance stationary and invertible if this model obeys the usual stationarity and invertibility constraints on the polynomials $1-\sum_{j=1}^{p} \gamma_{j} L^{j}$ and $1+\sum_{j=1}^{q} \delta_{j} L^{j}$. The implication is that the autocorrelation function of $\left\{g_{t}\right\}$ and the corresponding unconditional variance can be found using standard results on covariance stationary linear processes. Further, following the initial draft of this paper, Streett (2000) has shown that the above model has a unique stationary distribution.

In our numerical work we enforce covariance stationarity and invertibility constraints on the GLARMA representation of $\left\{g_{t}\right\}$. This is carried out by parameterising the model in terms of the partial autocorrelations $\left\{\rho_{j}, j=1, \ldots, p\right\}$ and the inverse partial autocorrelations $\left\{\bar{\rho}_{j}, j=1, \ldots, q\right\}$ (e.g. Barndorff-Nielsen and Schou (1973) and Jones (1987)). The corresponding likelihood is maximised using analytic first derivatives and the BHHH algorithm ${ }^{2}$.

\footnotetext{
${ }^{2} \mathrm{~A}$ plain $\mathrm{BHHH}$ method was used mapping the real variables being maximised into partial autocorrelations and inverse partial autocorrelations using the transform $x /(1+|x|)$. No interventions or numerical problems were encountered. The prediction decomposition of the GLARMA likelihood function is initialised by setting $g_{0}, \ldots, g_{-p+1}, v_{0}, \ldots, v_{-q+1}$ to zero.
} 
Using an AIC model selection rule we have chosen a GLARMA(3,1) model for the activity dataset. The estimated parameters and diagnostic statistics are given in Table 2 . Notice that the likelihood for this model is much higher, and the diagnostics much better behaved, than for the previous models fitted for activity given in Table 1. However, the estimated coefficients for the lagged values of direction and (to a lesser extent) large moves have not changed very much. Table 2 shows that both $L_{t-1}$ and $L_{t-2}$ are marginally significant. Both their corresponding parameter estimates and standard errors are not very stable across different GLARMA models. The direction variables are much more important in this context and these are estimated precisely and are not very sensitive to the parameterisation of the dynamics we use.

\begin{tabular}{|c|ccl|}
\hline Variable & Coef. & StR. Err. \\
\hline$\rho_{1}$ & 0.99988 & \\
$\rho_{2}$ & -0.649 & & \\
$\rho_{3}$ & -0.289 & & \\
$\bar{\rho}_{1}$ & -0.986 & & \\
$D_{t-1}$ & -0.103 & $(.012)$ & \\
$D_{t-2}$ & -0.0575 & $(.013)$ & \\
$L_{t-1}$ & -0.198 & $(.085)$ & \\
$L_{t-2}$ & 0.231 & $(.095)$ & \\
\hline $\mathrm{Q}$ & $T \sum_{j=1}^{Q} r_{j}^{2}$ & & Log-like $=-81,927$ \\
\hline 20 & 21.88 & $(31.41)$ & \\
100 & 92.9 & $(124.3)$ & \\
1,500 & 1,447 & $(1,591)$ & \\
\hline
\end{tabular}

Table 2: Estimation for the activity using a GLARMA model. Parameter estimates of the fitted GLARMA model, using a maximum likelihood criteria and the (3) error term. The figures in brackets are the standard errors on the regressors computed using the GLARMA model. Model order selected using AIC. $r_{j}$ denotes the series correlation coefficient at lag $j$ for the the standardised residuals $v_{t}$. The figures in brackets are corresponding 95 percentage points on the $\chi_{Q}^{2}$ distribution.

The estimated parameters suggest a great deal of memory in the activity series. Of course, we have imposed stationarity on this process and so it will be important to model the possibility that this series is non-stationary. More realistically we need a more intricate model of activity which takes into account intra-day, intra-week and month effects on the series. Work on this topic is reported in the next section. 


\subsection{The direction of price changes}

An important feature of our decomposition is that we are now able to focus on a model of the directions of the price changes, given that the price has changed: $\operatorname{Pr}\left(D_{t} \mid \mathcal{F}_{t-1}, A_{t}=1\right)$.

Again we will use an autologistic model, but this time the outcome variable will live on the support $\{-1,1\}$, rather than $\{0,1\}$. After testing out insignificant explanatory variables we end up with directions and large-direction as the only information of significance, where large direction is given by

$$
L D_{t}=\left(S_{t}-A_{t}\right) D_{t}
$$

Furthermore, let

$$
T_{t}=\sup _{s_{1}, \ldots, s_{k}}\left\{\begin{array}{rr}
s_{1}<t ; & A_{s_{1}}=1 \\
s_{2}<s_{1} ; & A_{s_{2}}=1 \\
\vdots & \\
s_{k}<s_{k-1} ; & A_{s_{k}}=1
\end{array}\right\} .
$$

The $T_{t}$ vector is $k \times 1$ and contains the times at which the last $k$ active prices occurred trades which moved the price level. We call this concept of a time scale "activity time". We found that this measurement of time to be extremely significant statistically.

Then $D_{T_{t}}$ will be a vector of the last $k$ price changes different from 0 . We refer to the $i$ - th element of this vector as $D_{T_{t}, i}$, which is the $i-t h$ last price move that has been observed, standing at time $t$. A simple example of this is $D_{T_{t}, 1}$ which is the sign of the last price movement different from 0 .

This gives us an autologistic model for

$$
\operatorname{Pr}\left(D_{t}=1 \mid \mathcal{F}_{t-1}, A_{t}=1\right)=p\left(\theta_{t}^{D}\right), \quad \text { where } \quad p\left(\theta_{t}^{D}\right)=\frac{\exp \left(\theta_{t}^{D}\right)}{1+\exp \left(\theta_{t}^{D}\right)},
$$

and so

$$
\operatorname{Pr}\left(D_{t}=-1 \mid \mathcal{F}_{t-1}, A_{t}=1\right)=\frac{1}{1+\exp \left(\theta_{t}^{D}\right)} .
$$

By going from general to specific we find that direction has only very short memory, see Table 3. From the estimated parameters we see that the process $D_{t}$ is strongly meanreverting (in activity time) reflecting the observed directions. An implication of this fitted model is that the dynamics generating the directions seems symmetrical, although there are more up directions than down ones - we will see down movements are typically bigger than up moves which compensates for this feature.

The lagged values of $D_{t}$ and $L D_{t}$ seem reasonably straightforward. If the price moved on the last trade then there is a large chance that this movement will be reversed if there is 
an active trade. If it moved by two ticks this probability of a reversal is reduced, although not by a great deal. On the other hand, if the last active trade was two periods ago the chance of a reversal is not very much different from 0.5 .

The really interesting variables are the overwhelmingly significant, but quite modest in effect, variables $\left\{D_{T_{t}, i}\right\}$. These relate current directions to the last active trade which appears at a random number of trades ago. That is they work in activity time not in trading time. They suggest that the sign of the last active trade has a sustained effect on the probability of an up or down. A simple example is that, at whatever lag in trading time, if the last price movement was down then there is a slightly higher probability of a reversal than a non-reversal.

An interesting effect, which is only marginally significant, is the $\sum_{i=11}^{20} D_{T_{t}, i}$ variable. If this variable is larger than zero then the series has tended to have a lot of up price movements and not many downs. So this is recording the presence of local trends in the price. It suggests this has a mildly positive effect on the direction process.

Our fitted model has some empirical failures. The diagnostic checks in Table 3 suggest we are slightly failing the check on serial correlation for this model. This failure should be put in some perspective. When we fit a model with just a constant (the directions are i.i.d. Bernoulli - an implication of the model suggested by Rogers and Zane (1998)) the log-likelihood is -22,966, and the Box-Pierce statistic at 20 lags is 9, 173 . An alternative model is a simple autologistic in activity time - that is regressing just on $\left\{D_{T_{t}, i}, i=1,2, \ldots, 10\right\}$, then we have a log-likelihood of $-20,416$ and a Box-Pierce of only 21. That model has reasonably good diagnostics but not an enormous amount of explanatory power. A simple alternative is to run a logistic regression using $\left\{D_{t-i}, i=1,2, \ldots, 20\right\}$, which is modelling directions using data ordered in transaction time, rather than activity time. This has a quite a high log-likelihood of $-18,419$ but its Box-Pierce at 20 lags is 688. Hence this model has the opposite problem - being able to predict many of the directions but failing dramatically the diagnostics. It seems very hard to remove this model failure when we only use the concept of transaction time. The introduction of activity time seems essential for this type of process.

The economic meaning of this fitted model is that the directions are mostly generated by bid/ask bounce - see, for a review of empirical work on this topic, Campbell, Lo, and MacKinlay (1997, pp. 99-107). That is people buying shares from market makers have to pay higher prices for them than those selling them to the market makers (an elegant model of bid and ask dynamics is given by Hasbrouck (1999)). Sequences of no 


\begin{tabular}{|c|cc||c|cc|}
\hline Variable & Estimate & Std. Err. & Variable & Estimate & Std. Err. \\
\hline$D_{t-1}$ & -2.192 & .043 & $L D_{t-1}$ & 0.629 & .180 \\
$D_{t-2}$ & -0.672 & .033 & $L D_{t-2}$ & -0.506 & .160 \\
$D_{t-4}$ & 0.296 & .030 & $L D_{t-3}$ & -0.837 & .200 \\
$D_{t-5}$ & 0.395 & .033 & $L D_{t-5}$ & -0.625 & .191 \\
$D_{t-6}$ & 0.337 & .034 & $D_{T_{t}, 1}$ & -0.403 & .038 \\
$D_{t-7}$ & 0.249 & .034 & $D_{T_{t}, 2}$ & 0.307 & .036 \\
$D_{t-8}$ & 0.233 & .034 & $D_{T_{t}, 3}$ & $-0 . .069$ & .031 \\
$D_{t-9}$ & 0.141 & .034 & $D_{T_{t}, 5}$ & -0.056 & .027 \\
$D_{t-10}$ & 0.073 & .031 & $D_{T_{t}, 8}$ & 0.059 & .027 \\
$D_{t-13}$ & -0.086 & .030 & $D_{T_{t}, 10}$ & -0.059 & .027 \\
$D_{t-14}$ & -0.067 & .029 & $\sum_{j=11}^{20} D_{T_{t}, j}$ & 0.025 & .010 \\
$D_{\left(T_{t}, 1\right)-1}$ & 0.315 & .032 & Const. & -0.053 & .067 \\
$D_{\left(T_{t}, 3\right)-1}$ & -0.087 & .030 & & & \\
$D_{\left(T_{t}, 4\right)-1}$ & -0.134 & .033 & & & \\
$D_{\left(T_{t}, 5\right)-1}$ & -0.105 & .033 & & & \\
$D_{\left(T_{t}, 6\right)-1}$ & -0.128 & .033 & & & \\
$D_{\left(T_{t}, 7\right)-1}$ & -0.154 & .032 & & & \\
$D_{\left(T_{t}, 8\right)-1}$ & -0.106 & .030 & & & \\
\hline $\mathrm{Q}$ & $T \sum_{j=1}^{Q} r_{j}^{2}$ & & & & \\
\hline 20 & 39.25 & $(31.41)$ & & & \\
100 & 101.4 & $(124.3)$ & & & \\
1,500 & 1,510 & $(1,591)$ & & & \\
\hline
\end{tabular}

Table 3: Estimation results for the direction of active trade using an autologistic model. The figures in brackets are the standard errors on the regressors computed using the autologistic model. $r_{j}$ denotes the series correlation coefficient at lag $j$ for the the standardised residuals $u_{t}$. The figures in brackets correspond to 95 percentage points on the $\chi_{Q}^{2}$ distribution.

price movements are thought of as a series of consecutive buys (or sells) by the market makers. A price movement could reflect either a change in the efficient price or, more likely, a sell (or buy) by the market maker. As this buying and selling around the efficient price dominates in magnitude the actual large movements in the efficient price, it will automatically generate very strong negative autocorrelation in the directions sequences. That is changes in the traded price are almost certainly reversed.

\subsection{The size of price movements}

This section is devoted to constructing a model for $\operatorname{Pr}\left(S_{t} \mid \mathcal{F}_{t-1}, A_{t}=1, D_{t}\right)$. As we have noted above this is a process on the strictly positive integers. Although the sample size is around 33,000, there are only 261 of these which are not one. Hence, we have to use 
quite simple models in this part of the paper as this dataset is not very informative about the dynamics of the size of price movements. We will use a negative binomial based GLARMA process for large movements $S_{t}-1$. Recall the negative binomial (NegBin) is a generalisation of the Poisson, allowing overdispersion (see, for example, Johnson, Kotz, and Kemp (1992, pp. 204-5)). The model will have

$$
\operatorname{Pr}\left(S_{t}=s_{t} \mid \mathcal{F}_{t-1}, A_{t}=1, D_{t}\right)=\frac{\Gamma\left(\alpha+s_{t}-1\right)}{\Gamma(\alpha)\left(s_{t}-1\right) !}\left(\frac{\alpha}{\mu_{t}+\alpha}\right)^{\alpha}\left(\frac{\mu_{t}}{\mu_{t}+\alpha}\right)^{s_{t}-1}
$$

implying

$$
\begin{aligned}
E\left(S_{t} \mid \mathcal{F}_{t-1}\right) & =1+\mu_{t} \\
\operatorname{Var}\left(S_{t} \mid \mathcal{F}_{t-1}\right) & =\alpha\left(\frac{\mu_{t}}{\mu_{t}+\alpha}\right) /\left(\frac{\alpha}{\mu_{t}+\alpha}\right)^{2}=\mu_{t}+\frac{1}{\alpha} \mu_{t}^{2} .
\end{aligned}
$$

Notice that $\mu_{t}$ will be typically very small and so $\mu_{t}^{2}$ is mostly tiny. As $\alpha$, the overdispersion parameter, goes to infinity so NegBin approaches a conditional Poisson model. Here we allow $\mu_{t}=\exp \left(\theta_{t}^{S}\right)$ where

$$
\theta_{t}^{S}=x_{t}^{\prime} \beta+g_{t}, \quad \text { and } \quad g_{t}=\sum_{j=1}^{p} \gamma_{j} g_{t-j}+\sigma v_{t}+\sigma \sum_{j=1}^{q} \delta_{j} v_{t-j},
$$

where $x_{t}$ will include $D_{t}$ and elements of $\mathcal{F}_{t-1}$. We define

$$
v_{t}=\frac{\left\{\left(S_{t-1}-1\right)-\mu_{t-1}\right\}}{\sqrt{\mu_{t-1}+\frac{1}{\alpha} \mu_{t}^{2}}} .
$$

as our basic parametric model ${ }^{3}$. The NegBin distribution was selected as it is simple and familiar.

The model was fitted using a maximum likelihood estimator, selecting $p, q$ using AIC. The resulting estimated model is detailed in Table 4 . The most interesting feature is that the direction of the current price change and the preceding are significant and all have negative coefficients. This rejects, quite significantly, two hypotheses of symmetry. Firstly big price movements tend to be preceded by non-symmetric falls in the price (directions being negative). This is the familiar dynamic leverage effect (see Nelson (1991)) and so its presence is not surprising.

The second form of non-symmetry is a contemporaneous one and is simply dependent on the significance of $D_{t}$ (not its lags). The negative sign associated with it suggests

\footnotetext{
${ }^{3}$ In foreign exchange markets the tick size tends to be smaller compared to the bid-ask spread. However, many writers have observed that there is a tendency for prices to cluster on "natural numbers" such as integers. Our model would have to be altered to allow for such a characteristic, but from a methodological viewpoint this raises no new issues.
} 
big falls are more common than big rises. This implies the unconditional distribution of returns should be skewed with a longer left hand tail (if $D_{t}$ had a zero coefficient then the implied unconditional distribution of returns would be symmetric). This is counterbalanced by the fact that the average value of $S_{t} D_{t}$ is positive, which suggests the market trends upwards over time due to the predominance of small positive movements (over small negative movements), but tends to fall back sometimes with quite large falls.

The above non-symmetry of the unconditional distribution has not been found in previous work on this type of data. This is perhaps not surprising as the t-statistic on it is only around 8 and so will not be found unless it is very directly tested. It is, however, important. Our results suggest large movements (movements of more than one tick) downwards are, on average, around twice as large as large movements upwards.

\begin{tabular}{|c|cc||c|cc|}
\hline Variable & Coef. & StR. Err. & Variable & Coef. & StR. Err. \\
\hline Const & -5.140 & $(.138)$ & $\sigma$ & 0.180 & \\
$\rho_{1}$ & 0.998 & & $\rho_{2}$ & -0.343 & \\
$\bar{\rho}_{1}$ & -0.816 & & $D_{t}$ & -0.320 & $(.070)$ \\
$D_{t-1}$ & -0.394 & $(.095)$ & $D_{t-3}$ & -0.299 & $(.112)$ \\
$D_{\left(T_{t}, 1\right)-5}$ & -0.121 & $(.067)$ & $D_{\left(T_{t}, 1\right)-6}$ & -0.206 & $(.064)$ \\
$\alpha$ & 0.0713 & & & & \\
\hline $\mathrm{Q}$ & $T \sum_{j=1}^{Q} r_{j}^{2}$ & & & \multicolumn{2}{|c|}{ Log-like $=-1,478$} \\
\hline 20 & 31.88 & $(31.41)$ & $E\left(v_{t}\right)=-.001$ & & \\
100 & 85.39 & $(124.3)$ & $\operatorname{Var}\left(v_{t}\right)=1.207$ & & \\
1,500 & 1,743 & $(1,591)$ & & & \\
\hline
\end{tabular}

Table 4: Estimation for the NegBin based GLARMA(3,1) model of the excess price movements. Variable is the explanatory variable. Std. Err. denotes the standard deviation. The figures in brackets are the standard errors on the regressors computed using the GLARMA model. Model order selected using AIC. $r_{j}$ denotes the series correlation coefficient at lag $j$ for the the standardised residuals $u_{t}$. The figures in brackets are corresponding 95 percentage points on the $\chi_{Q}^{2}$ distribution.

Judging from Table 4 the NegBin based GLARMA model is failing slightly as the variance of the residuals is slightly bigger than one. This could be due to misspecification in the construction of $\left\{\mu_{t}\right\}$, or a mild distributional failure in our choice of the NegBin model. In the next section we will condition on a wider information set and so may hope to remove this problem by using a more subtle version of $\left\{\mu_{t}\right\}$.

To put the performance of this model structure in context we can compare its fit to a constant which gives a log-likelihood of $-1,628$ and a Box-Pierce statistic (using 20 lags) of 1,762 . When we take out the GLARMA structure completely, leaving just explanatory 
variables, the log-likelihood is $-1,602$ and the Box-Pierce is 2,065. The other extreme is were we drop all the explanatory variables and leave simply the $\operatorname{GLARMA}(2,1)$ model. This has a log-likelihood of -1,502 and Box-Pierce of 16.6. Thus we can see that it is the time series modelling aspect of this particular model which dominates the fitting of this series.

\section{Comments}

\subsection{Roll's model of bid-ask bounce}

In an insightful paper, Roll (1984) proposed a simple measure for the effective bid-ask spread. The measure given for the spread was simply

$$
2 \times \sqrt{-\operatorname{Cov}\left(Z_{t}, Z_{t-1}\right)}
$$

where Cov denotes the unconditional covariance. This was based on the observation that market efficiency should guarantee that the covariance between efficient prices should be 0 and that the actual observed covariance is due only to the bid-ask spread. The model proposed in Roll's paper is probably too simplistic to tell the whole story about serial correlation but what is important to note is that a large amount of the first-order serial covariance is due to bid-ask effects something which is easily captured by our model for the directions $D_{t}$.

Roll's measure of spread is an unconditional one, but our analysis suggests a generalization. We argue that one could infer spread from

$$
2 \sqrt{-\operatorname{Cov}\left(Z_{t}, Z_{t-1} \mid \mathcal{F}_{t-2}\right)}
$$

a conditional correlation. As $\mathcal{F}_{t-2}$ varies, so does the implied spread. In particular it may widen if we have observed a sequence of active price movements.

\subsection{Predictive distributions}

\subsubsection{Multi-step prediction}

A crucial use of our model structure is to produce multi-step ahead predictions of asset price movements. This can be expressed in two basic ways: (i) predictions of the $(s+1)-$ periods ahead price movements, (ii) predictions of the asset price levels $(s+1)$-periods ahead. We first of all deal with the former. 


\subsubsection{Predicting price movements}

The object of interest is $\operatorname{Pr}\left(Z_{t+s} \mid \mathcal{F}_{t-1}\right)$, which is

$$
\begin{aligned}
\operatorname{Pr}\left(Z_{t+s} \mid \mathcal{F}_{t-1}\right) & =\sum_{Z_{t}} \ldots \sum_{Z_{t+s-1}} \operatorname{Pr}\left(Z_{t}, \ldots, Z_{t+s} \mid \mathcal{F}_{t-1}\right) \\
& =\sum_{Z_{t}} \ldots \sum_{Z_{t+s-1}} \prod_{j=0}^{s} \operatorname{Pr}\left(Z_{t+j} \mid \mathcal{F}_{t-1+j}\right)
\end{aligned}
$$

In our model $Z_{t}$ lives on the integers which makes complete enumeration of these quantities impossible. We can respond to this in two ways - by using simulation or by truncating the state space of $Z_{t}$. For small values of $s$ the latter probably is the most effective, while for long horizons simulations would seem perfectly satisfactory for most purposes.

For $s$ very large the multi-step ahead forecast distribution of price movements will approach the unconditional distribution of our fitted model ${ }^{4}$. Although this is of little economic meaning it can be a useful diagnostic check on the fitted model.

\subsubsection{Predicting price levels}

Computing analytically predicted price levels can be carried out using similar arguments to those given above for any value of $s$.

The calculations become intricate when $s$ is large as there are many groups of price changes which achieve the same terminal price. Hence, in practical work the best way of proceeding is by the use of simulation. Hence given $\mathcal{F}_{t-1}$ we simulate the process $N$ times and count the number of simulated prices which fall on particular lattice points. As our model is extremely easy to simulate from this can be carried out for very large values of $N$ (in the simulations discussed below $N=10,000)$ even if $s$ is large.

Table 5 shows the centre of the two-step ahead forecast distribution of price moves based on different histories. The histories are made as a simple as possible. We assume that (i) trading has not been moving the prices for some time, that is $\mathcal{F}^{A}=$ $(1,1,0, \ldots, 0)$ (ii) the last two trades before we forecast both moved the prices, that is $\mathcal{F}^{D}=(?, ?,-1,1,-1)$ where we will replace ?,? by moves of one tick in various directions. We then tabulate the forecast distribution over all possible one tick price changes in the last two periods. Column one shows the impact of two down movements having just been observed and this is seen to give an increased probability for moving one tick up. Column four has the opposite observation, namely two up movements. Here we have the

\footnotetext{
${ }^{4}$ We should note that this makes an assumption that the $Z_{t}$ process is stationary, which we have not proven.
} 
opposite result that the probability of moving down is increased. The middle two columns corresponds to "bid-ask bouncing". This has decreased the probability of moving away from the current price level. From all the columns it is seen that the predominant behaviour is mean reversion of one tick size and that the last directions are important in determining how likely a price reversal is. This implies that, with the given history, when we have seen a movement of two one ticks down(up) after two trades the price will still be a least one down(up) with probability 0.990(0.991) and at least two down(up) with probability $0.707(0.756)$.

In Table 5 we also give the 10-step ahead forecast. In this case we get that when we have seen a movement of two one ticks down(up) after ten trades the price will still be a least two down(up) with probability $0.779(0.809)$.

\begin{tabular}{|cc||cccc|}
\hline tick moves & no. of trades & $\{-1,-1\}$ & $\{-1,1\}$ & $\{1,-1\}$ & $\{1,1\}$ \\
\hline \hline-3 & 2 & 0.00077 & 0.00017 & 0.00094 & 0.00020 \\
& 10 & 0.0027 & 0.0019 & 0.0025 & 0.0029 \\
\hline-2 & 2 & 0.00409 & 0.00164 & 0.00907 & 0.00834 \\
& 10 & 0.0108 & 0.0097 & 0.0104 & 0.0106 \\
\hline-1 & 2 & 0.05440 & 0.05176 & 0.24669 & 0.23538 \\
& 10 & 0.1512 & 0.1268 & 0.1982 & 0.1698 \\
\hline 0 & 2 & 0.64761 & 0.66028 & 0.68549 & 0.69373 \\
& 10 & 0.6141 & 0.6115 & 0.6211 & 0.6104 \\
\hline 1 & 2 & 0.28354 & 0.27661 & 0.05655 & 0.05833 \\
& 10 & 0.2006 & 0.2262 & 0.1505 & 0.1850 \\
\hline 2 & 2 & 0.00894 & 0.00913 & 0.00103 & 0.00330 \\
& 10 & 0.0120 & 0.0140 & 0.0095 & 0.0117 \\
\hline 3 & 2 & 0.00007 & 0.00036 & 0.00006 & 0.00050 \\
& 10 & 0.0023 & 0.0033 & 0.0024 & 0.0022 \\
\hline
\end{tabular}

Table 5: The Table show the simulated probabilities for having moved $x$ ticks, after 2 or 10 trades, given the history. $\mathcal{F}^{A}=(1,1,0, \ldots, 0)$ and $\mathcal{F}^{D}=(?, ?,-1,1,-1)$. ?,? is given in the top row of the Table. The estimated probabilities are based on $N=10,000$ simulations.

\subsection{Previous work}

\subsubsection{Conditional multinomial models}

In a recent highly stimulating paper Russell and Engle (1998) have suggested modelling price movements using a conditional multinomial distribution. Their paper can be viewed as a time series extension of a probit (Russell and Engle (1998) prefer to work with logistic 
functions rather than probit ones) analysis of transaction data proposed by Hausman, Lo, and MacKinlay (1992). Here we will discuss their work and its relationship to our own. We will initially abstract our discussion from the time series feature of the model and so we will write $Y_{t}$ to denote the indicator for the movements which we will assume live only on $-2,-1,0,1,2$. So if the movement is 1 , then $Y_{t}=(0,0,0,1,0)^{\prime}$, while if it were -1 then $Y_{t}=(0,1,0,0,0)^{\prime}$. We suppose we use some regressors $X_{t}$ to model the changing probabilities of these movements. In practice $X_{t}$ will depend upon some features of the filtration of $Y_{t}, \mathcal{F}_{t}^{Y}=\sigma\left(Y_{s}, s \leq t\right)$.

At this level, there is only one loss of generality (and information) compared to our decomposition - price movements have to live on a small finite grid (mainly due to parsimony). Next Russell and Engle (1998) use a multinomial logit structure (see e.g. McFadden (1984, Section 3.4)).

$$
\operatorname{Pr}\left(Y_{t}=i \mid X_{t}\right)=p_{i}\left(\theta_{t}\right), \quad i=-2,-1,0,1,2
$$

where $\theta_{t}=\left(\theta_{-2 t}, \theta_{-1 t}, \theta_{0 t}, \theta_{1 t}, \theta_{2 t}\right)^{\prime}=X_{t} \beta$ and

$$
p_{i}\left(\theta_{t}\right)=\frac{\exp \left(\theta_{i, t}\right)}{1+\sum_{j=-2}^{2} \exp \left(\theta_{j, t}\right)}, \quad i=-2,-1,0,1,2 .
$$

In practice this structure is not identified and so constraints are placed on $X_{t} \beta$. A typical situation would be to define $\theta_{0 t}=0$ for all $t$, a solution followed by Russell and Engle (1998).

The important step in Russell and Engle (1998) is to define a vector generalised linear autoregressive moving average (VGLARMA) type structure on $\theta_{t}^{*}=\left(\theta_{-2 t}, \theta_{-1 t}, \theta_{1 t}, \theta_{2 t}\right)^{\prime}$, feeding in lagged values of $\left\{y_{t}\right\}$ using the variable given by (3). In particular if they define $v_{t}=\left(v_{1 t}, v_{2 t}, v_{3 t}, v_{4 t}\right)^{\prime}$ with

$$
v_{i t}=\frac{I\left(Y_{t}=i\right)-p_{i t}}{\sqrt{p_{i t}\left(1-p_{i t}\right)}},
$$

and

$$
\theta_{t}^{*}=\alpha^{*}+g_{t}
$$

then model this system as

$$
g_{t}=\sum_{j=1}^{p} \gamma_{j} g_{t-j}+\sigma v_{t}+\sigma \sum_{j=1}^{q} \delta_{j} v_{t-j}
$$

where $\alpha^{*}$ is a vector, while $\left\{\gamma_{j}\right\}, \sigma$ and $\left\{\delta_{j}\right\}$ are $4 \times 4$ matrices. The only a priori constraint we might place on this structure is that $\sigma$ should be lower triangular for identification. 
Overall we can see that our analysis is quite closely related to that of Hausman, Lo, and MacKinlay (1992) and Russell and Engle (1998). Our goals are the same, although the technology that we use is very different. Our main advantages are: parsimony, easy interpretability via the decomposition and options for extensions.

\subsubsection{Hasbrouck's truncation model}

Hasbrouck (1999) introduced a dynamic model for the evolution of the bid and ask price of quotation data. Let $\mu_{t}$ denote the theoretical efficient price in the market and let $\alpha_{t}, \sigma_{t}$ represent the ask and bid costs respectively. Then Hasbrouck argued for a structure where the bid price is Floor $\left(\mu_{t}-\alpha_{t}\right)$ and the ask price is Ceiling $\left(\mu_{t}-\sigma_{t}\right)$. Here the Floor function rounds down to the nearest tick and Ceiling rounds up. Related papers include Bollerslev and Melvin (1994) and Harris (1994).

The Hasbrouck (1999) bid/ask model is not immediately applicable to transaction data, but the principle of using a continuous time model which is then truncated in some way is potentially useful if combined (perhaps) with the Hausman, Lo, and MacKinlay (1992) static model of clustering.

\subsection{Conditioning on signs}

In some recent work, carried out independently from our own, Granger (1998) has emphasised the potential importance of modelling separately the direction (sign) and the size of stochastic processes. Typically he models these two variables independently, while we emphasise the sequential nature of our decomposition — which is empirically vital for our problem and more general. Abstracting from that detail, we can see that our analysis can be seen within his framework when we condition on the activity, $A_{t}$, being one.

\subsection{Explanatory variables}

\subsubsection{Deterministic seasonality}

It may be that the activity, directions and size series are influenced by deterministic seasonal patterns for we know these patterns influence $N(u)$ the rate at which transactions occur in calendar time - see, for example, Rydberg and Shephard (2000). It is a straightforward task to include this information within our model, allowing seasonality to influence any or all of the sub-models for activity, direction and size. No new issues are raised by this and we will give empirical results on this in a later subsection. 


\subsubsection{Exogenous variables}

Our modelling framework allows some very simple extensions which will be potentially enriching. Suppose in addition to the price movements $\left\{Z_{t}\right\}$ we have a sequence of other information sets such as volume and place of trade. Let us write these additional variables as $\left\{Y_{t}\right\}$, then we can do a prediction decomposition, using the extended filtration $\mathcal{F}_{t}^{z, y}=$ $\sigma\left(Z_{s}, Y_{s}: s \leq t\right)$, to give

$$
\begin{aligned}
f\left(Z_{1}, Y_{1}, \ldots, Z_{n}, Y_{n} \mid \mathcal{F}_{0}^{z, y}\right) & =\prod_{t=1}^{n} f\left(Z_{t}, Y_{t} \mid \mathcal{F}_{t-1}^{z, y}\right) \\
& =\prod_{t=1}^{n} f\left(Y_{t} \mid \mathcal{F}_{t-1}^{z, y}\right) \operatorname{Pr}\left(Z_{t} \mid Y_{t}, \mathcal{F}_{t-1}^{z, y}\right) \\
& =\prod_{t=1}^{n} \operatorname{Pr}\left(Z_{t} \mid \mathcal{F}_{t-1}^{z, y}\right) f\left(Y_{t} \mid Z_{t}, \mathcal{F}_{t-1}^{z, y}\right)
\end{aligned}
$$

where the second stage of decomposition can be useful if we can find a sensible model for $f\left(Y_{t} \mid Z_{t}, \mathcal{F}_{t-1}^{z, y}\right)$ and we can allow $Y_{t}$ to enrich the decomposition of the price innovation process. The third stage decomposition can also be the focus of attention as it allows lagged information to improve our predictions of future price movements given the history of the $Y_{t}$ process.

The above decomposition suggests that there are potentially two interesting densities for $\left\{Z_{t}\right\}$ to investigate further: $\operatorname{Pr}\left(Z_{t} \mid \mathcal{F}_{t-1}^{z, y}\right)$ and $\operatorname{Pr}\left(Z_{t} \mid Y_{t}, \mathcal{F}_{t-1}^{z, r}\right)$. The first is a pure forecast while the second allows contemporaneous explanatory variables to enter (see Engle, Hendry, and Richard (1983) and Hendry (1995, Ch. 5)). Tables 6 and 7 give results for the activity and direction series when we condition on lagged variables. The variables we use in this exercise are the logarithm of the volume traded and the logarithm of the number of seconds (plus one) elapsing before the trade. In addition we use dummy variables to denote the hour, day of the week and month of the year in which the trade takes place. Finally, we sometimes use two trending variables: range (a standardised version of the time index $t$ ) and quadr which is simply the square of range. These trends are used as a parsimonious representation of the monthly seasonal pattern. Further, we have tried using the log of the actual price level of the IBM stock price, but this always tested out in our empirical work. Notice that in Tables 6 and 7 (as well as other tables given below) many of these fixed explanatory variables do not appear, which is due to them being tested out as insignificant.

The empirical model for directions, reported in Table 7, is interesting as it completely tests out the effect of durations on the prediction of directions, while the influence of lagged volume is very small and almost all seasonal effects are irrelevant. 


\begin{tabular}{|c|c|c|c|c|c|}
\hline Variable & Estimate & Std. Err. & Variable & Estimate & Std. Err. \\
\hline Const. & -1.509 & $(.045)$ & Range & 0.126 & $(.031)$ \\
\hline$\rho_{1}$ & 0.9998 & & $\log (\text { dur })_{t-1}$ & 0.028 & $(.005)$ \\
\hline$\rho_{2}$ & -0.668 & & $\log (\text { dur })_{t-2}$ & -0.018 & $(.005)$ \\
\hline$\rho_{3}$ & -0.252 & & $\log (\text { dur })_{t-3}$ & -0.020 & $(.005)$ \\
\hline $\bar{\rho}$ & -0.987 & & $\log (\text { dur })_{t-4}$ & -0.020 & $(.005)$ \\
\hline$L_{t-1}$ & -0.219 & $(.085)$ & $\log (\text { dur })_{t-5}$ & -0.017 & $(.005)$ \\
\hline$L_{t-2}$ & 0.235 & $(.095)$ & $\log (\text { dur })_{t-6}$ & -0.016 & $(.005)$ \\
\hline$D_{t-1}$ & -0.101 & $(.012)$ & $\log (\text { dur })_{t-8}$ & -0.009 & $(.005)$ \\
\hline$D_{t-2}$ & -0.058 & $(.013)$ & $\sum_{j=11}^{20} \log (\text { dur })_{t-j}$ & -0.004 & $(.002)$ \\
\hline & & & $\sum_{j=21}^{30} \log (\text { dur })_{t-j}$ & -0.005 & $(.002)$ \\
\hline Q & \multicolumn{2}{|l|}{$T \sum_{j=1}^{Q} r_{j}^{2}$} & & \multicolumn{2}{|c|}{ Log-like $=-81839$} \\
\hline 20 & 19.06 & $(31.41)$ & & & \\
\hline 100 & 87.2 & $(124.3)$ & & & \\
\hline 1500 & 1437 & $(1591)$ & & & \\
\hline
\end{tabular}

Table 6: Estimation for the activity including lagged $\log ($ duration +1$)$ and the $\log ($ volume $)$ (which tests out). Std. Err. denotes the standard deviation.

This is not the case when we look at the activity series, which is sensitive to many lags of durations. This is perhaps not surprising as the activity series is connected to volatility and so one would expected them to be influenced by other activity type series. The range variable is a trend variable, which we interpret as a monthly seasonal type variable rather than a typical trend as we only have a year of data. We used this range variable rather than a full set of monthly seasonal for reasons of parsimony.

An interesting feature for both the activity and direction series is that lagged volume and duration variables are sometimes statistically significant but not overwhelmingly so. Instead lagged data on previous price movements completely dominate the fit of these two models.

In Tables 8 and 9 we report the estimated activity and direction processes using contemporaneous volumes and durations. For the activity series current durations have a very dramatic positive impact on activity. A smaller impact is made by volume. In addition hourly seasonal effects are now significant.

The quantitative effect of this is quite large. Activity is affected positively by both volume and duration (see Table 8). In particular if the duration is high then this increases the chance that the price will move at the next trade, while if volume if high the same thing happens.

When we look at direction (see Table 9) we see that again both variables have signifi- 


\begin{tabular}{|c|c|c|c|c|}
\hline Exp. Var. & estimate & Std. Err. & Exp. Var. & estimate Std. Err. \\
\hline$D_{t-1}$ & -2.192 & .043 & $L D_{t-1}$ & 0.620 \\
\hline$D_{t-2}$ & -0.671 & .033 & $L D_{t-2}$ & -0.506 \\
\hline$D_{t-4}$ & 0.298 & .030 & $L D_{t-3}$ & -0.851 \\
\hline$D_{t-5}$ & 0.395 & .033 & $L D_{t-5}$ & -0.626 \\
\hline$D_{t-6}$ & 0.337 & .034 & $D_{T_{t}, 1}$ & -0.400 \\
\hline$D_{t-7}$ & 0.248 & .034 & $D_{T_{t}, 2}$ & 0.301 \\
\hline$D_{t-8}$ & 0.232 & .034 & $D_{T_{t}, 3}$ & -0.071 \\
\hline$D_{t-9}$ & 0.139 & .034 & $D_{T_{t}, 5}$ & -0.062 \\
\hline$D_{t-10}$ & 0.072 & .031 & $\log (\mathrm{vol})_{t-1}$ & -0.030 \\
\hline$D_{t-13}$ & -0.083 & .030 & $\log (\mathrm{vol})_{t-2}$ & 0.021 \\
\hline$D_{t-14}$ & -0.067 & .029 & $\sum_{j=11}^{20} D_{T_{t}, j}$ & 0.012 \\
\hline$D_{\left(T_{t}, 1\right)-1}$ & 0.312 & .032 & April & 0.140 \\
\hline$D_{\left(T_{t}, 3\right)-1}$ & -0.086 & .030 & Const. & -0.070 \\
\hline$D_{\left(T_{t}, 4\right)-1}$ & -0.133 & .033 & & \\
\hline$D_{\left(T_{t}, 5\right)-1}$ & -0.103 & .033 & & \\
\hline$D_{\left(T_{t}, 6\right)-1}$ & -0.127 & .033 & & \\
\hline$D_{\left(T_{t}, 7\right)-1}$ & -0.152 & .032 & & \\
\hline$D_{\left(T_{t}, 8\right)-1}$ & -0.106 & .030 & & \\
\hline $\mathrm{Q}$ & $T \sum_{j=1}^{Q} r_{j}^{2}$ & & & Log-like $=-17938$ \\
\hline 20 & 47.78 & $(31.41)$ & & \\
\hline 100 & 110.4 & $(124.3)$ & & \\
\hline 1500 & 1513 & $(1591)$ & & \\
\hline
\end{tabular}

Table 7: Estimation for the direction including lagged durations (which test out) and volume. Improvement in the log-likelihood for introducing these variables is 9. The figures in brackets are the standard errors on the regressors computed using the autologistic model. $r_{j}$ denotes the series correlation coefficient at lag $j$ for the the standardised residuals $u_{t}$. The figures in brackets are corresponding 95 percentage points on the $\chi_{Q}^{2}$ distribution.

cant impact. High durations reduce the chance that the price movement will be upwards, while high volume increases the chance of an up movement.

The estimated NegBin based GLARMA model for the large variable $S_{t}-1$ is reported in Table 10 using lagged durations and volume. The AIC measure selected a $\operatorname{GLARMA}(2,1)$ structure. Interestingly the effect of lagged durations is modest, with its influence being played out over just two lags. Both of the estimated coefficients have t-statistics of only around 3. The lagged volume variables have a bigger impact, with longer lags than that given in the tables having positive but insignificant effects on the large variable. Importantly the range and quadr variable is taken as estimating the seasonal component of the process. This is significant, which is unsurprising given the size variable is, like the activity variable, a kind of volatility measure. Also the time series dependence in the 


\begin{tabular}{|c|rr||c|rr|}
\hline Variable & estimate & Std. Err. & Variable & estimate & Std. Err. \\
\hline Const. & -1.460 & 0.045 & Range & 0.157 & 0.028 \\
$\rho_{1}$ & 0.9998 & & $L D_{t-1}$ & -0.215 & 0.086 \\
$\rho_{2}$ & -0.624 & & $L D_{t-2}$ & 0.368 & 0.097 \\
$\rho_{3}$ & -0.245 & & $D_{t-1}$ & -0.107 & 0.012 \\
$\bar{\rho}$ & -0.986 & & $D_{t-2}$ & -0.048 & 0.013 \\
$10-11$ & -0.082 & 0.039 & $\log (\mathrm{vol})_{t}$ & 0.064 & 0.004 \\
$11-12$ & -0.154 & 0.043 & $\log (\mathrm{dur})_{t}$ & 0.368 & 0.005 \\
$12-13$ & -0.145 & 0.046 & $\log (\mathrm{dur})_{t-1}$ & 0.070 & 0.005 \\
$13-14$ & -0.173 & 0.047 & $\log (\mathrm{dur})_{t-2}$ & -0.013 & 0.005 \\
$14-15$ & -0.128 & 0.044 & $\log (\mathrm{dur})_{t-3}$ & -0.020 & 0.005 \\
$\sum_{j=11}^{20} \log (\mathrm{dur})_{t-j}$ & -0.009 & 0.002 & $\log (\mathrm{dur})_{t-4}$ & -0.024 & 0.005 \\
$\sum_{j=21}^{30} \log (\mathrm{dur})_{t-j}$ & -0.009 & 0.002 & $\log (\mathrm{dur})_{t-5}$ & -0.022 & 0.005 \\
& & & $\log (\mathrm{dur})_{t-6}$ & -0.023 & 0.005 \\
& & & $\log (\mathrm{dur})_{t-8}$ & -0.015 & 0.005 \\
\hline $\mathrm{Q}$ & $T \sum_{j=1}^{Q} r_{j}^{2}$ & & & Log-like $=-79,022$ \\
\hline 20 & 39.07 & $(31.41)$ & & & \\
100 & 115.0 & $(124.3)$ & & & \\
\hline 1,500 & 1,490 & $(1,591)$ & & & \\
\hline
\end{tabular}

Table 8: Estimation for the activity including contemporaneous and lagged $\log ($ duration +1$)$ and the $\log ($ volume $)$. Std. Err. denotes the standard deviation.

GLARMA model has been reduced quite considerably by the presence of the explanatory variables.

Overall lagged explanatory variables improve the likelihood function by around 24, which is modest given we have included seven new explanatory variables in the fitted model. The diagnostic checks on the fitted model have improved, especially at short lags, but the model still suffers from slight over-dispersion suggesting some improvement could be gained from fitting a more complicated model than the NegBin structure we have used.

Table 11 shows the fitted model for the size variable using contemporaneous volumes and durations, in addition to lagged data and seasonal effects. The table shows that contemporaneous explanatory variables have a very significant effect on the large movement variables $S_{t}-1$. The volume variable has a very large positive impact on the chance that an active variable moves the price by more than one tick. The t-statistic on current volume is around 20, which is by far the largest of any of the significant variables we have found for the large movement variable. Interestingly the presence of current volume reduces the impact of lagged volume and removes the need to have daily seasonals and the quadratic trend (monthly seasonal). All that remains of these deterministic seasonals 


\begin{tabular}{|c|cc||c|cc|}
\hline Variable & Estimate & Std. Err. & Variable & Estimate & Std. Err. \\
\hline$D_{t-1}$ & -2.173 & .043 & $L D_{t-1}$ & 0.638 & .181 \\
$D_{t-2}$ & -0.663 & .033 & $L D_{t-2}$ & -0.490 & .161 \\
$D_{t-4}$ & 0.298 & .030 & $L D_{t-3}$ & -0.830 & .199 \\
$D_{t-5}$ & 0.395 & .033 & $L D_{t-5}$ & -0.607 & .192 \\
$D_{t-6}$ & 0.334 & .034 & $D_{T_{t}, 1}$ & -0.412 & .038 \\
$D_{t-7}$ & 0.241 & .034 & $D_{T_{t}, 2}$ & 0.301 & .036 \\
$D_{t-8}$ & 0.225 & .034 & $D_{T_{t}, 3}$ & -0.073 & .031 \\
$D_{t-9}$ & 0.135 & .034 & $D_{T_{t}, 5}$ & -0.062 & .027 \\
$D_{t-10}$ & 0.071 & .031 & $\log (\operatorname{vol})_{t}$ & 0.087 & .008 \\
$D_{t-13}$ & -0.092 & .030 & $\log (\operatorname{vol})_{t-1}$ & -0.039 & .009 \\
$D_{t-14}$ & -0.069 & .029 & $\log (\mathrm{dur})_{t}$ & -0.110 & .010 \\
$D_{\left(T_{t}, 1\right)-1}$ & 0.316 & .032 & $\sum_{j=11}^{20} D_{T_{t}, j}$ & 0.012 & .005 \\
$D_{\left(T_{t}, 3\right)-1}$ & -0.087 & .030 & April & 0.150 & .050 \\
$D_{\left(T_{t}, 4\right)-1}$ & -0.135 & .033 & Const. & 0.082 & .039 \\
$D_{\left(T_{t}, 5\right)-1}$ & -0.103 & .033 & & & \\
$D_{\left(T_{t}, 6\right)-1}$ & -0.126 & .033 & & & \\
$D_{\left(T_{t}, 7\right)-1}$ & -0.155 & .032 & & & \\
$D_{\left(T_{t}, 8\right)-1}$ & -0.106 & .030 & & & \\
\hline $\mathrm{Q}$ & $T \sum_{j=1}^{Q} r_{j}^{2}$ & & & & \\
\hline 20 & 47.76 & $(31.41)$ & & & \\
100 & 112.3 & $(124.3)$ & & & \\
1,500 & 1,540 & $(1,591)$ & & & \\
\hline
\end{tabular}

Table 9: Estimation for the direction including lagged and contemporaneous durations and volume. Improvement in the log-likelihood for introducing the contemporaneous variables is 101. The figures in brackets are the standard errors on the regressors computed using the autologistic model. $r_{j}$ denotes the series correlation coefficient at lag $j$ for the the standardised residuals $u_{t}$. The figures in brackets are corresponding 95 percentage points on the $\chi_{Q}^{2}$ distribution.

is the range variable, which we should interprete as saying big moves occur towards the end of the year. However, this effect is not very significant.

The contemporaneous duration variable also has a positive impact while at one lag the effect is reversed. We do not understand this effect.

The presence of these new explanatory variables cleans up the serial dependence structure in the data, for now the Box-Pierce statistics are satisfactory. Further the amount of over-dispersion in the fitted model is modest. 


\begin{tabular}{|c|cc||c|cc|}
\hline Variable & Coef. & StR. Err. & Variable & Coef. & StR. Err. \\
\hline Const & -5.546 & $(.152)$ & $\sigma$ & 0.175 & \\
Tues & 0.683 & $(.184)$ & Wednes & 0.489 & $(.222)$ \\
Range & 0.391 & $(.124)$ & Quadr & -0.342 & $(.114)$ \\
$\rho_{1}$ & 0.996 & & $\rho_{2}$ & -0.264 & \\
$\bar{\rho}_{1}$ & -0.805 & & $\alpha$ & 0.077 & \\
$D_{t}$ & -0.347 & $(.073)$ & $D_{t-1}$ & -0.488 & $(.100)$ \\
$D_{t-3}$ & -0.328 & $(.116)$ & & & \\
$D_{\left(T_{t}, 1\right)-6}$ & -0.183 & $(.068)$ & & & \\
$\log (\mathrm{dur})_{t-1}$ & -0.163 & $(.068)$ & & & \\
$\log (\operatorname{vol})_{t-1}$ & 0.144 & $(.052)$ & $\log (\operatorname{vol})_{t-2}$ & 0.110 & $(.045)$ \\
\hline $\mathrm{Q}$ & $T \sum_{j=1}^{Q} r_{j}^{2}$ & & & Log-like $=-1,454$ \\
\hline 20 & 27.05 & $(31.41)$ & $E\left(v_{t}\right)=-.002$ & & \\
100 & 119.2 & $(124.3)$ & $\operatorname{Var}\left(v_{t}\right)=1.166$ & & \\
1,500 & 1,393 & $(1,591)$ & & & \\
\hline
\end{tabular}

Table 10: Estimation for the NegBin based GLARMA(2,1) model of the excess price movements $\left(S_{t}-1\right)$ including lagged $\log ($ duration +1$)$ and the $\log ($ volume $)$. Std. Err. denotes the standard deviation. The figures in brackets are the standard errors on the regressors computed using the GLARMA model. Model order selected using AIC. $r_{j}$ denotes the series correlation coefficient at lag $j$ for the the standardised residuals $v_{t}$. The figures in brackets are corresponding 95 percentage points on the $\chi_{Q}^{2}$ distribution.

\section{Conclusions}

In this paper we have proposed a decomposition of the price movements of trade-by-trade datasets. The decomposition means we have to model sequentially price activity, direction of moves and size of moves. Each modelling exercise is straightforward and interpretable. A number of extensions of the modelling framework are possible, including the use of relevant weakly exogenous variables.

When combined with a good model for the times between trades this analysis provides a complete model for the evolution of prices in real time.

Interesting open issues include: (i) modelling of two or more asset prices simultaneously, (ii) using trade data on the same stock but collected on different exchanges.

\section{Acknowledgments}

Tina Rydberg thanks the Danish National Research Council for their financial support through a post-doctoral fellowship. Neil Shephard thanks the ESRC for financial support through the grant R00023839. Both authors are grateful for support from The Centre 


\begin{tabular}{|c|cc||c|cc|}
\hline Variable & Coef. & StR. Err. & Variable & Coef. & StR. Err. \\
\hline Const & -5.710 & $(.120)$ & $\sigma$ & 0.149 & \\
Range & 0.315 & $(.118)$ & $\bar{\rho}_{1}$ & -0.830 & \\
$\rho_{1}$ & 0.996 & & $\rho_{2}$ & -0.411 & \\
$D_{t}$ & -0.291 & $(.070)$ & $D_{t-1}$ & -0.296 & $(.095)$ \\
$\sum_{j=1}^{6} D_{\left(T_{t}, 1\right)-j}$ & -0.195 & $(.061)$ & & & \\
$\log (\mathrm{dur})_{t}$ & 0.179 & $(.066)$ & $\log (\mathrm{dur})_{t-1}$ & -0.196 & $(.064)$ \\
$\log (\mathrm{vol})_{t}$ & 0.622 & $(.045)$ & $\alpha$ & 0.161 & \\
$\sum_{j=1}^{2} \log (\mathrm{vol})_{t-j}$ & 0.081 & $(.027)$ & $\sum_{j=3}^{5} \log (\mathrm{vol})_{t-j}$ & 0.062 & $(.023)$ \\
\hline $\mathrm{Q}$ & $T \sum_{j=1}^{Q} r_{j}^{2}$ & & & Log-like $=-1,355$ \\
\hline 20 & 19.21 & $(31.41)$ & $E\left(v_{t}\right)=-.001$ & & \\
100 & 111.6 & $(124.3)$ & $\operatorname{Var}\left(v_{t}\right)=1.171$ & & \\
1,500 & 1,587 & $(1,591)$ & & & \\
\hline \multicolumn{7}{|r}{} \\
\hline
\end{tabular}

Table 11: Estimation for the NegBin based GLARMA(2,1) model of the excess price movements $\left(S_{t}-1\right)$ including contemporaneous and lagged $\log ($ duration +1$)$ and the $\log$ (volume). Std. Err. denotes the standard deviation. The figures in brackets are the standard errors on the regressors computed using the GLARMA model. Model order selected using AIC. $r_{j}$ denotes the series correlation coefficient at lag $j$ for the the standardised residuals $u_{t}$. The figures in brackets are corresponding 95 percentage points on the $\chi_{Q}^{2}$ distribution.

for Analytical Finance, Aarhus, Denmark. The authors are grateful for the comments from the participants at the conference on "Econometrics and Financial Time Series" at the Isaac Newton Institute, Cambridge University, 12-16 October 1998 where the details of our decomposition were first presented. We thank Richard Spady, Rob Engle, Frank Gerhard, Clive Bowsher and Jeff Russell for various helpful conversations on these issues. The computations were carried out using software written in Ox (Doornik (2001)).

\section{References}

Barndorff-Nielsen, O. E. and G. Schou (1973). On the reparameterization of autoregressive models by partial autocorrelations. Journal of Multivariate Analysis 3, 408-419.

Bollerslev, T. and M. Melvin (1994). Bid-ask spreads in the foreign exchange market: an empirical analysis. Journal of International Economics 36, 355-72.

Campbell, J. Y., A. W. Lo, and A. C. MacKinlay (1997). The Econometrics of Financial Markets. Princeton, New Jersey: Princeton University Press.

Cox, D. R. (1958). The regression analysis of binary sequences (with discussion). Journal of the Royal Statistical Society, Series B 20, 215-42. 
Cox, D. R. and E. J. Snell (1989). The Analysis of Binary Data (2 ed.). London: Chapman \& Hall.

Darolles, S., C. Gourieroux, and G. Le Fol (2000). Intra-day transaction price dynamics. Annales d'Economie et Statistique 60, 207-238.

Doornik, J. A. (2001). Ox: Object Oriented Matrix Programming, 3.0. London: Timberlake Consultants Press.

Engle, R. F. (2000). The econometrics of ultra-high frequency data. Econometrica 68, $1-22$.

Engle, R. F., D. F. Hendry, and J. F. Richard (1983). Exogeneity. Econometrica 51, $277-304$.

Engle, R. F. and J. Russell (2002). High-frequency and transaction data. In Y. AitSahalia and L. P. Hansen (Eds.), Handbook of Financial Econometrics. NorthHolland. Forthcoming.

Engle, R. F. and J. R. Russell (1998). Forecasting transaction rates: the autoregressive conditional duration model. Econometrica 66, 1127-1162.

Ghysels, E. and J. Jasiak (1998). GARCH for irregularly spaced financial data: the ACD-GARCH model. Studies in Nonlinear Dynamics and Econometrics 2, 133149.

Granger, C. W. J. (1998). Comonotonicity. Lecture to Econometrics and Financial Time Series Workshop, Isaac Newton Institute for Mathematical Sciences, Cambridge University.

Harris, L. E. (1994). Minimum price variation, discrete bid-ask spreads and quotation sizes. Review of Financial Studies 7, 149-78.

Hasbrouck, J. (1999). The dynamics of discrete bid and ask quotes. Journal of Finance 54, 2109-2142.

Hausman, J., A. W. Lo, and A. C. MacKinlay (1992). An ordered probit analysis of transaction stock prices. Journal of Financial Economics 31, 319-30.

Hendry, D. F. (1995). Dynamic Econometrics. Oxford: Oxford University Press.

Johnson, N. L., S. Kotz, and A. W. Kemp (1992). Univariate Discrete Distributions (2 ed.). New York: John Wiley. 
Jones, M. C. (1987). Randomly choosing parameters for the stationary and invertibility region of autoregressive-moving average models. Applied Statistics 36, 134-138.

Manganelli, S. (2001). Duration, volume and volatility impact of trades. Unpublished paper: European Central Bank.

McCullagh, P. and J. A. Nelder (1989). Generalized Linear Models (2 ed.). London: Chapman \& Hall.

McFadden, D. L. (1984). Qualitative response models. In Z. Griliches and M. Intriligator (Eds.), The Handbook of Econometrics, Volume 2, pp. 1395-1457. North-Holland.

Meddahi, N., E. Renault, and B. Werker (1998). Modelling high frequency data in continuous time. Unpublished paper: CIRANO, CRDE, Montreal University.

Nelson, D. B. (1991). Conditional heteroskedasticity in asset pricing: a new approach. Econometrica 59, 347-370.

Rogers, L. C. G. and O. Zane (1998). Designing and estimating models of high frequency data. Unpublished paper: Department of Mathematics, University of Bath. Presented at Workshop on Mathematical Finance, University of Bremen, Germany, February.

Roll, R. (1984). A simple implicit measure of the efficient bid-ask spread in an efficient market. Journal of Finance 39, 1127-1139.

Russell, J. R. and R. F. Engle (1998). Econometric analysis of discrete-valued, irregularly-spaced financial transactions data using a new autoregressive conditional multinomial models. Unpublished paper: Graduate School of Business, University of Chicago. Presented at Second international conference on high frequency data in finance, Zurich, Switzerland, April.

Rydberg, T. H. and N. Shephard (2000). A modelling framework for the prices and times of trades made on the NYSE. In W. J. Fitzgerald, R. L. Smith, A. T. Walden, and P. C. Young (Eds.), Nonlinear and Nonstationary Signal Processing, pp. 217 246. Cambridge: Isaac Newton Institute and Cambridge University Press.

Shephard, N. (1994). Autoregressive based generalized linear models. Unpublished paper: Nuffield College, Oxford. Presented at the Econometric Society World Congress, Tokyo, 1995.

Streett, S. (2000). Some observation driven models for time series. Unpublished Ph.D. thesis, Department of Statistics, Colorado State University. 